\title{
Weak Krull-Schmidt for Infinite Direct Sums of Cyclically Presented Modules over Local Rings
}

\author{
Afshin Amini (*) - Babak Amini (*) - Alberto Facchini $(* *)\left({ }^{a}\right)$
}

\section{Introduction.}

There is a surprising analogy between the behavior of direct sums of uniserial modules over an arbitrary ring and the behavior of direct sums of cyclically presented modules over local rings. The reason of this analogy lies mainly in the fact that both the endomorphism ring of a uniserial module over an arbitrary ring and the endomorphism ring of a cyclically presented module over a local ring are either local or exactly have two maximal ideals whose residue rings are division rings. This allows us to give a complete description of the direct-sum decompositions of a module into uniserial submodules or into cyclically presented submodules (when the base ring is local) exactly using two invariants. These two invariants are the epigeny class and the monogeny class in the case of uniserial modules [4], and are the epigeny class and the lower part in the case of cyclically presented modules over local rings [1]. In both cases, a Weak Krull-Schmidt Theorem for finite direct sums holds. It is now natural to try to see which further parts of the theory of uniserial modules developed in the last decade also holds for cyclically presented modules over local rings.

(*) Indirizzo dell'A.: Department of Mathematics, College of Sciences, Shiraz University, Shiraz 71454, Iran.

E-mail: aamini@shirazu.ac.ir bamini@shirazu.ac.ir

(**) Indirizzo dell'A.: Dipartimento di Matematica Pura e Applicata, Università di Padova, Via Trieste 63, 35121 Padova, Italy.

E-mail: facchini@math.unipd.it

(a) Partially supported by Ministero dell'Università e della Ricerca (Prin 2005 "Perspectives in the theory of rings, Hopf algebras and categories of modules") and by Gruppo Nazionale Strutture Algebriche e Geometriche e loro Applicazioni of Istituto Nazionale di Alta Matematica.

2000 Mathematics Subject Classification. Primary 15A33, 16D70. Secondary $16 \mathrm{~L} 30$. 
The first natural question is to see whether the Weak Krull-Schmidt Theorem holds for infinite direct sums as well. For uniserial modules, this question was answered in three papers. First, Dung and Facchini [3] individuated a possible "pathology", given by the so-called non-quasismall modules, as a possible source of difficulties. Secondly, Puninski [11] proved the existence of non-quasismall modules. And thirdly, Př́hoda [9] finally determined the correct form of the Weak Krull-Schmidt Theorem for infinite direct sums of uniserial modules. In this paper, we answer the corresponding question for infinite direct sums of cyclically presented modules over a local ring, that is, we prove a Weak Krull-Schmidt Theorem that holds for infinite direct sums of cyclically presented modules over local rings (Theorem 3.1). The case of cyclically presented modules turns out to be much simpler than that of uniserial modules, essentially because cyclically presented modules are finitely generated, hence small, so that the pathology of non-quasismall modules cannot appear in this setting.

Another natural question is whether a direct summand of a direct sum of uniserial modules (cyclically presented modules over a local ring) is still a direct sum of uniserial (cyclically presented, respectively) modules; see [5, Problems 9 and 10, p. 268]. For infinite direct sums of uniserial modules, the answer is negative, as was proved by Puninski in [10, Theorem 6.3]. The same holds for cyclically presented modules over a local ring, because the example given by Puninski in [11, Proposition 8.1] of a uniserial domain $R$ with a non-zero non-invertible element $r$ and a direct-sum decomposition $K \oplus(R / r R)^{\left(\aleph_{0}\right)} \cong(R / r R)^{\left(\aleph_{0}\right)}$, shows not only the existence of a uniserial module $K$ that is not quasi-small, but also that there is a countably generated uniserial direct summand $K$ of a direct sum of cyclically presented modules over the local ring $R$ that is not a direct sum of cyclically presented modules. Thus this second question has a negative answer both in the case of uniserial modules and in the case of cyclically presented modules over local rings. Here is another example. In [10, Theorem 6.3], there is an example of a pure projective module $P$ over a uniserial coherent ring that is not direct sum of indecomposable modules. Over a uniserial ring, every finitely presented module is a direct sum of cyclically presented modules. Therefore, $P$ is another example of a module that is not a direct sum of cyclically presented modules, but is a direct summand of a direct sum of cyclically presented modules.

A third natural question is therefore whether a direct summand of a direct sum of finitely many uniserial modules (cyclically presented modules over a local ring) is still a direct sum of uniserial (cyclically presented, respectively) modules. In the finite case, Př́hoda [8] showed that every 
direct summand of a direct sum of finitely many uniserial modules is still a direct sum of finitely many uniserial modules. The general case of direct summands of direct sums of finitely many cyclically presented modules over a local ring is still open.

Direct summands of direct sums of cyclically presented modules are usually called RD-projective modules in the literature. Here "RD" stands for "relatively divisible". As we have seen above, there exist countably generated indecomposable RD-projective modules $K$ over local rings that are not direct sums of cyclically presented modules and RD-projective modules $P$ over local rings that are not direct sums of indecomposable modules. Nevertheless, every RD-projective module over a commutative local ring is a direct sum of cyclically presented modules [12, Corollary 2]. However, every RD-projective module over an arbitrary ring is a direct sum of countably generated RD-projective modules. In order to see this, notice that if a module $M$ is a direct sum of $c$-generated modules, where $c$ is any fixed infinite cardinal, then so is every direct summand of $M[5$, Theorem 2.47]. For $c=\aleph_{0}$, we get that every RD-projective module over an arbitrary ring, not necessarily local, is a direct sum of countably generated RD-projective modules. Therefore every RD-projective module over an arbitrary ring is a direct sum of direct summands of direct sums of countably many cyclically presented modules.

As we have already said, in this paper we completely solve the first question posed above, showing that the Weak Krull-Schmidt Theorem holds for infinite direct sums of cyclically presented modules over local rings (Theorem 3.1). In the last section of the paper, we study the monoid of all isomorphism classes of direct sums of cyclically presented modules over a local ring.

This paper is the natural generalization of our previous article [1], from the finite case to the infinite one. Throughout the paper, we consider unitary right modules over an associative ring with identity. For a ring $R$, $J(R)$ and $U(R)$ will be the Jacobson radical and the set of all invertible elements of $R$, respectively.

\section{Preliminary results.}

Recall that a right module over a ring $R$ is said to be cyclically presented if it is isomorphic to $R / a R$ for some $a \in R$. The endomorphism ring of a non-zero cyclically presented module $R / a R$ is canonically isomorphic to $E / a R$, where $E:=\{r \in R \mid r a \in a R\}$ is the idealizer of $a R$ and the right 
ideal $a R$ of $R$ is a two-sided ideal in the subring $E$ of $R$. The following result is proved in [1, Theorem 2.1].

THEOREM 2.1. Let a be a non-zero non-invertible element of a local ring $R$, let $E$ be the idealizer of aR in $R$, and let $E / a R$ be the endomorphism ring of the cyclically presented right $R$-module $R / a R$. Set

$$
I:=\{r \in R \mid r a \in a J(R)\} \text { and } K:=J(R) \cap E .
$$

Then $I$ and $K$ are completely prime two-sided ideals of $E$ containing $a R$, the union $(I / a R) \cup(K / a R)$ is the set of all non-invertible elements of $E / a R$, and every proper right ideal of $E / a R$ and every proper left ideal of $E / a R$ is contained either in I/aR or in K/aR. Moreover, exactly one of the following two conditions holds:

(a) Either the ideals $I$ and $K$ are comparable, so that $E / a R$ is a local ring with maximal ideal $(I / a R) \cup(K / a R)$, or

(b) The ideals $I$ and $K$ are not comparable, $J(E / a R)=(I \cap K) / a R$, and $(E / a R) / J(E / a R)$ is canonically isomorphic to the direct product of the two division rings $E / I$ and $E / K$.

From now on, for a non-projective cyclically presented module $U=R / a R$ over a local ring $R$, we use the notation $\mathcal{I}_{U}, \mathcal{K}_{U}$ for the two ideals $I / a R, K / a R$ of $\operatorname{End}_{R}(U)$ described in Theorem 2.1.

Recall that two modules $A$ and $B$ have the same epigeny class (notation $\left.[A]_{e}=[B]_{e}\right)$, if there exist an epimorphism $A \rightarrow B$ and an epimorphism $B \rightarrow A$; see [4]. Notice that, if $a, b$ are elements of a local ring $R$, then $[R / a R]_{e}=[R / b R]_{e}$ if and only if there exist $u, v \in U(R)$ with $u a \in b R$ and $v b \in a R$, or, equivalently, if there exist $u, v \in U(R)$ and $r, s \in R$ with $u a=b r$ and $v b=a s$ (because any epimorphism $R / a R \rightarrow R / b R$ is induced by left multiplication by some invertible element of $R$ ). If $R / a R$ and $R / b R$ are two cyclically presented modules over a local ring $R$, we say that $R / a R$ and $R / b R$ have the same lower part, and write $[R / a R]_{l}=[R / b R]_{l}$, if there exist $u, v \in U(R)$ and $r, s \in R$ with $a u=r b$ and $b v=s a$, equivalently, if there exist $r, s \in R$ such that $r b R=a R$ and $s a R=b R$ (see [1]). The unique cyclically presented module, up to isomorphism, with the same epigeny class as 0 is 0 , and the unique cyclically presented module, up to isomorphism, with the same epigeny class as $R_{R}$ is $R_{R}$. Similarly for the lower part.

The significance of these concepts for cyclically presented modules over local rings is highlighted by the fact that any cyclically presented module is uniquely determined by its lower part and its epigeny class. 
Lemma 2.2 [1, Proposition 4.2]. Let $U$ and $V$ be cyclically presented modules over a local ring $R$. Then $U \cong V$ if and only if $[U]_{l}=[V]_{l}$ and $[U]_{e}=[V]_{e}$.

For the reader's convenience, we collect in the rest of this section some results that will be used repeatedly in the sequel. The proof of Lemma 2.3 follows from [1, Lemma 5.2], Lemma 2.4 follows from [5, Corollary 4.6], and Lemma 2.5 follows from Theorem 2.1 .

LEMMA 2.3. Let $V, V^{\prime}$ be cyclically presented modules over a local ring $R$. Suppose that there exists a cyclically presented module $U$ such that $[U]_{l}=[V]_{l}$ and $[U]_{e}=\left[V^{\prime}\right]_{e}$. Then there exists a cyclically presented module $U^{\prime}$ such that $V \oplus V^{\prime} \cong U \oplus U^{\prime}$. Moreover, $\left[U^{\prime}\right]_{l}=\left[V^{\prime}\right]_{l}$ and $\left[U^{\prime}\right]_{e}=[V]_{e}$.

LEMMA 2.4 (Cancellation property). Let $A, B$ be right modules over a local ring $R$ and $U$ a cyclically presented right $R$-module such that $A \oplus U \cong B \oplus U$. Then $A \cong B$.

LEMMA 2.5. Let U be a non-projective cyclically presented module over an arbitrary local ring $R$.

(a) Iff and $g$ are two endomorphisms of $U$ such that $f \in \mathcal{I}_{U} \backslash \mathcal{K}_{U}$ and $g \in \mathcal{K}_{U} \backslash \mathcal{I}_{U}$, then $f+g$ is an automorphism.

(b) Conversely, suppose that $f_{1}, \ldots, f_{n}$ are $n$ endomorphisms of $U$, none of which is an automorphism. If $f_{1}+\cdots+f_{n}$ is an automorphism, then there exist two distinct indices $i, k=1,2, \ldots, n$ such that $f_{i} \in \mathcal{I}_{U} \backslash \mathcal{K}_{U}$ and $f_{k} \in \mathcal{K}_{U} \backslash \mathcal{I}_{U}$.

LEMMA 2.6. Let $A$ and $B$ be non-zero cyclically presented right modules over a local ring $R$ with $A$ non-projective.

(a) If there exist homomorphisms $\alpha: A \rightarrow B$ and $\beta: B \rightarrow A$ with $\beta \alpha \notin \mathcal{I}_{A}$, then $[A]_{l}=[B]_{l}$.

(b) If there exist homomorphisms $\alpha: A \rightarrow B$ and $\beta: B \rightarrow A$ with $\beta \alpha \notin \mathcal{K}_{A}$, then $[A]_{e}=[B]_{e}$.

The proof of Lemma 2.6 follows from the fact that, for any $a, b \in R$, every homomorphism $R / a R \rightarrow R / b R$ is induced by left multiplication by some $r \in R$ with $r a R \subseteq b R$. 


\section{Arbitrary families of cyclically presented modules over local rings.}

Consider two arbitrary families of cyclically presented modules $\left\{U_{i} \mid i \in I\right\}$ and $\left\{V_{j} \mid j \in J\right\}$ over a local ring $R$. The main result of this section says that $\bigoplus_{i \in I} U_{i} \cong \bigoplus_{j \in J} V_{j}$ if and only if the families $\left\{U_{i} \mid i \in I\right\}$ and $\left\{V_{j} \mid j \in J\right\}$ have the same lower parts and the same epigeny classes. This fact was proved for finite families of cyclically presented modules in [1].

Theorem 3.1. Let $\left\{U_{i} \mid i \in I\right\}$ and $\left\{V_{j} \mid j \in J\right\}$ be two families of nonzero cyclically presented modules over a local ring $R$. Then $\bigoplus_{i \in I} U_{i} \cong \bigoplus_{j \in J} V_{j}$ if and only if there exist two bijections $\sigma, \tau: I \rightarrow J$ such that $\left[U_{i}\right]_{l}=\left[V_{\sigma(i)}\right]_{l}$ and $\left[U_{i}\right]_{e}=\left[V_{\tau(i)}\right]_{e}$ for every $i \in I$.

Proof. Suppose that there are two bijections $\sigma, \tau: I \rightarrow J$ such that $\left[U_{i}\right]_{l}=\left[V_{\sigma(i)}\right]_{l}$ and $\left[U_{i}\right]_{e}=\left[V_{\tau(i)}\right]_{e}$ for every $i \in I$. We have to show that $\bigoplus_{i \in I} U_{i} \cong \bigoplus_{j \in J} V_{j}$

The symmetric group $S_{I}$ consisting of all bijections $I \rightarrow I$ acts on the set $I$ in a natural way. Let $C$ be the cyclic subgroup of $S_{I}$ generated by $\tau^{-1} \sigma \in S_{I}$. Then $C$ acts on the set $I$. For every element $i \in I$ let

$$
[i]=\left\{\left(\tau^{-1} \sigma\right)^{z}(i) \mid z \in \mathbb{Z}\right\}
$$

denote the $C$-orbit of $i$. Let $\sigma([i])=\{\sigma(x) \mid x \in[i]\}$ be the image of the orbit $[i]$ via the bijection $\sigma$.

Fix an element $i \in I$. We claim that $\bigoplus_{k \in[i]} U_{k} \cong \bigoplus_{\ell \in \sigma([i])} V_{\ell}$. In order to prove the claim, set, for simplicity of notation, $i_{z}:=\left(\tau^{-1} \sigma\right)^{z}(i), j_{z}:=\sigma\left(i_{z}\right)$, $U_{z}:=U_{i_{z}}$ and $V_{z}:=V_{j_{z}}$ for every $z \in Z$. Hence if the orbit [ $\left.i\right]$ is infinite, then $\sigma([i])$ is infinite, and $U_{n}=U_{m}$ if and only if $n=m$. If the orbit [ $\left.i\right]$ is finite of cardinality $q$, then $\sigma([i])$ is finite of cardinality $q$, and $U_{n}=U_{m}$ if and only if $n \equiv m(\bmod q)$. Note that $\tau\left(i_{z}\right)=\tau\left(\tau^{-1} \sigma\right)^{z}(i)=\sigma\left(\tau^{-1} \sigma\right)^{z-1}(i)=$ $=\sigma\left(i_{z-1}\right)=j_{z-1}$. In this notation, the equality $\left[U_{i}\right]_{l}=\left[V_{\sigma(i)}\right]_{l}$ for every $i \in I$ implies that

$$
\left[U_{z}\right]_{l}=\left[V_{z}\right]_{l}
$$

for all $z \in Z$, and similarly the equality $\left[U_{i}\right]_{e}=\left[V_{\tau(i)}\right]_{e}$ implies that

$$
\left[U_{z}\right]_{e}=\left[V_{z-1}\right]_{e}
$$

for all $z \in Z$. 
We now prove that there are cyclically presented modules $X_{0}, X_{1}, \ldots$ and $Y_{1}, Y_{2}, \ldots$ satisfying the following properties for every integer $n \geq 1$ :

(a) $X_{n-1} \oplus Y_{n} \cong V_{n-1} \oplus V_{-n}$ and $X_{n} \oplus Y_{n} \cong U_{n} \oplus U_{-n}$;

(b) $\left[X_{n}\right]_{l}=\left[U_{n}\right]_{l}$ and $\left[X_{n}\right]_{e}=\left[U_{-n}\right]_{e}$;

(c) $\left[Y_{n}\right]_{l}=\left[V_{-n}\right]_{l}$ and $\left[Y_{n}\right]_{e}=\left[V_{n-1}\right]_{e}$.

The construction of the $X_{i}$ 's and the $Y_{j}$ 's is by induction, as follows. Set $X_{0}:=U_{0}$. Since $\left[U_{0}\right]_{l}=\left[V_{0}\right]_{l}$ and $\left[U_{0}\right]_{e}=\left[V_{-1}\right]_{e}$, there is a cyclically presented module $Y_{1}$ such that $V_{0} \oplus V_{-1} \cong X_{0} \oplus Y_{1}$, $\left[Y_{1}\right]_{l}=\left[V_{-1}\right]_{l}$ and $\left[Y_{1}\right]_{e}=$ $=\left[V_{0}\right]_{e}\left(\right.$ Lemma 2.3). Therefore, $\left[U_{1}\right]_{e}=\left[V_{0}\right]_{e}=\left[Y_{1}\right]_{e}$ and $\left[U_{-1}\right]_{l}=\left[V_{-1}\right]_{l}=$ $=\left[Y_{1}\right]_{l}$. Hence, again by Lemma 2.3 , there is a cyclically presented module $X_{1}$ such that $U_{1} \oplus U_{-1} \cong X_{1} \oplus Y_{1},\left[X_{1}\right]_{l}=\left[U_{1}\right]_{l}$ and $\left[X_{1}\right]_{e}=\left[U_{-1}\right]_{e}$. Thus $X_{0}$, $X_{1}, Y_{1}$ have the required properties.

Now suppose $n>1$ and that $X_{t}$ and $Y_{t}$ satisfying the required properties have already been constructed for every $t<n$. Since $\left[X_{n-1}\right]_{l}=$ $=\left[U_{n-1}\right]_{l}=\left[V_{n-1}\right]_{l}$ and $\left[X_{n-1}\right]_{e}=\left[U_{-n+1}\right]_{e}=\left[V_{-n}\right]_{e}$, by Lemma 2.3, there exists a cyclically presented module $Y_{n}$ such that $X_{n-1} \oplus Y_{n} \cong V_{n-1} \oplus V_{-n}$, $\left[Y_{n}\right]_{l}=\left[V_{-n}\right]_{l}$ and $\left[Y_{n}\right]_{e}=\left[V_{n-1}\right]_{e}$. From $\left[U_{-n}\right]_{l}=\left[V_{-n}\right]_{l}$ and $\left[U_{n}\right]_{e}=$ $=\left[V_{n-1}\right]_{e}$, it follows that $\left[Y_{n}\right]_{l}=\left[U_{-n}\right]_{l}$ and $\left[Y_{n}\right]_{e}=\left[U_{n}\right]_{e}$. Again by Lemma 2.3, there exists a cyclically presented module $X_{n}$ such that $X_{n} \oplus Y_{n} \cong U_{n} \oplus U_{-n},\left[X_{n}\right]_{l}=\left[U_{n}\right]_{l}$ and $\left[X_{n}\right]_{e}=\left[U_{-n}\right]_{e}$.

Suppose that the orbit $[i]$ is an infinite set. Then

$$
\begin{aligned}
\bigoplus_{k \in[i]} U_{k}=\bigoplus_{n \in \mathbb{Z}} U_{n} & =U_{0} \oplus\left(\bigoplus_{n \geq 1}\left(U_{n} \oplus U_{-n}\right)\right) \\
& \cong X_{0} \oplus\left(\bigoplus_{n \geq 1}\left(X_{n} \oplus Y_{n}\right)\right) \\
& =\bigoplus_{n \geq 1}\left(X_{n-1} \oplus Y_{n}\right) \\
& \cong \bigoplus_{n \geq 1}\left(V_{n-1} \oplus V_{-n}\right) \\
& =\bigoplus_{n \in \mathbb{Z}} V_{n}=\bigoplus_{\ell \in \sigma([i])} V_{\ell} .
\end{aligned}
$$

This proves the claim for the case of an infinite orbit [ $i]$.

In order to prove the claim in the case in which the orbit $[i]$ is a finite set with $q$ elements, that is, in order to prove that $\bigoplus_{z=0}^{q-1} U_{z} \cong \bigoplus_{z=0}^{q-1} V_{z}$, it suffices to apply (1), (2) and the Weak Krull-Schmidt Theorem for finite direct 
sums of cyclically presented modules over local rings [1, Theorem 5.3]. Thus the claim is true.

When the index $i$ runs over all the indices in $I$, we get that the orbits $[i]$ form a partition of $I$ into disjoint countable subsets $I=\bigcup_{i \in I}[i]$ and their images $\sigma([i])$ form a partition of $J$ into disjoint countable subsets $J=\bigcup_{i \in I} \sigma([i])$. From the claim we know that $\bigoplus_{k \in[i]} U_{k} \cong \bigoplus_{\ell \in \sigma([i])} V_{\ell}$ for every orbit $[i]$, so that $\bigoplus_{i \in I} U_{i} \cong \bigoplus_{j \in J} V_{j}$.

For the proof of the opposite implication in the statement of Theorem 3.1, we first prove the following auxiliary lemma.

LEMma 3.2. Let $M=\bigoplus_{j \in J} A_{j}$ be a direct sum of arbitrary modules $A_{j}$ over a local ring $R$. Suppose that $M=U \oplus B$, where $U$ is a non-zero cyclically presented R-module. Let $\pi_{U}: M=U \oplus B \rightarrow U, \pi_{k}: M=\bigoplus_{j \in J} A_{j} \rightarrow A_{k}$ be the structural projections and $\varepsilon_{U}: U \rightarrow M=U \oplus B, \varepsilon_{k}: A_{k} \rightarrow M=\bigoplus_{j \in J} A_{j}$ be
the embeddings. Then:

(a) If $U$ is non-projective, there exist two indices $i, k \in J$ such that $\pi_{U} \varepsilon_{i} \pi_{i} \varepsilon_{U} \in \mathcal{I}_{U} \backslash \mathcal{K}_{U}$ and $\pi_{U} \varepsilon_{k} \pi_{k} \varepsilon_{U} \in \mathcal{K}_{U} \backslash \mathcal{I}_{U}$.

(b) If $U \cong R_{R}$, there exists an index $k \in J$ such that $\pi_{U} \varepsilon_{k} \pi_{k} \varepsilon_{U}$ is an automorphism of $U$.

Proof. As $U_{R}$ is finitely generated, there are $j_{1}, \ldots, j_{n} \in J$ such that $U \subseteq A_{j_{1}} \oplus \cdots \oplus A_{j_{n}}$. Therefore, for any $j \in J \backslash\left\{j_{1}, \ldots, j_{n}\right\}, \pi_{j}(U)=0$. Set $C:=\underset{j \neq j_{1}, \ldots, j_{j}}{\bigoplus} A_{j}$, so that $M=A_{j_{1}} \oplus \cdots \oplus A_{j_{n}} \oplus C$. If $\varepsilon_{U}, \pi_{U}, \varepsilon_{B}, \pi_{B}, \varepsilon_{j_{t}}, \pi_{j_{t}}$, $(t=1, \ldots, n)$ and $\varepsilon_{C}, \pi_{C}$ are the injections and the projections associated to the two direct-sum decompositions $M=U \oplus B=A_{j_{1}} \oplus \cdots \oplus A_{j_{n}} \oplus C$, then $\pi_{C}(U)=0$ and hence

$$
\begin{aligned}
1_{U}=\pi_{U} \varepsilon_{U} & =\pi_{U}\left(\varepsilon_{j_{1}} \pi_{j_{1}}+\cdots+\varepsilon_{j_{n}} \pi_{j_{n}}+\varepsilon_{C} \pi_{C}\right) \varepsilon_{U} \\
& =\pi_{U} \varepsilon_{j_{1}} \pi_{j_{1}} \varepsilon_{U}+\cdots+\pi_{U} \varepsilon_{j_{n}} \pi_{j_{n}} \varepsilon_{U}+\pi_{U} \varepsilon_{C} \pi_{C} \varepsilon_{U} \\
& =\pi_{U} \varepsilon_{j_{1}} \pi_{j_{1}} \varepsilon_{U}+\cdots+\pi_{U} \varepsilon_{j_{n}} \pi_{j_{n}} \varepsilon_{U} .
\end{aligned}
$$

Then we have two cases:

(a) If $U$ is non-projective, then by Lemma 2.5, there exist two indices $i, k \in\left\{j_{1}, \ldots, j_{n}\right\}$ such that $\pi_{U} \varepsilon_{i} \pi_{i} \varepsilon_{U} \in \mathcal{I}_{U} \backslash \mathcal{K}_{U}$ and $\pi_{U} \varepsilon_{k} \pi_{k} \varepsilon_{U} \in \mathcal{K}_{U} \backslash \mathcal{I}_{U}$.

(b) If $U \cong R_{R}$, then $\operatorname{End}_{R}(U) \cong R$ is a local ring, hence there exists an index $k \in\left\{j_{1}, \ldots, j_{n}\right\}$ such that $\pi_{U} \varepsilon_{k} \pi_{k} \varepsilon_{U}$ is an automorphism of $U$. 
We are now ready to prove the remaining implication in the proof of Theorem 3.1. We may suppose that $M=\bigoplus_{i \in I} U_{i}=\bigoplus_{j \in J} V_{j}$ with $U_{i}, V_{j}$ nonzero cyclically presented modules for every $i$ and $j$.

We construct the bijection $\sigma$, the existence of the other bijection $\tau$ can be proved exactly in the same way. For any non-zero cyclically presented module $U$, consider the two subsets $I(U)=\left\{i \in I \mid\left[U_{i}\right]_{l}=[U]_{l}\right\}$ of $I$ and $J(U)=\left\{j \in J \mid\left[V_{j}\right]_{l}=[U]_{l}\right\}$ of $J$. It is obvious that the $I(U)$ 's and the $J(U)$ 's, when $U$ ranges in all the non-zero cyclically presented modules, form a partition of $I$ and $J$, respectively.

In order to establish the existence of the bijection $\sigma$ between the lower parts of $\left\{U_{i} \mid i \in I\right\}$ and $\left\{V_{j} \mid j \in J\right\}$, it is sufficient to prove that the cardinalities $|I(U)|$ and $|J(U)|$ are equal for every non-zero cyclically presented module $U$.

Fix a non-zero cyclically presented module $U$. Suppose first that either $I(U)$ or $J(U)$ is a finite set. Without loss of generality we may assume $|I(U)| \leq|J(U)|$. Suppose that $|I(U)|<|J(U)|$. If $I(U)=\left\{i_{1}, \ldots, i_{n}\right\}$, let $\left\{j_{1}, \ldots, j_{n+1}\right\}$ be a subset of $J(U)$ of cardinality $n+1$. Write

$$
M=U_{i_{1}} \oplus \cdots \oplus U_{i_{n}} \oplus B=V_{j_{1}} \oplus \cdots \oplus V_{j_{n+1}} \oplus C,
$$

where

$$
B=\bigoplus_{i \neq i_{1}, \ldots, i_{n}} U_{i} \quad \text { and } \quad C=\bigoplus_{j \neq j_{1}, \ldots, j_{n+1}} V_{j} .
$$

We will show by induction on $n$ that the submodule $B$ has a cyclically presented direct summand $V$ isomorphic to $V_{j_{t}}$ for some $t=1, \ldots, n+1$, which gives us a contradiction because by Lemmas 2.6(a) and 3.2 there exists $i \in I \backslash\left\{i_{1}, \ldots, i_{n}\right\}$ with $\left[U_{i}\right]_{l}=[V]_{l}=\left[V_{j_{t}}\right]_{l}=[U]_{l}$.

If $U \cong R_{R}$, then $U_{i_{1}}, \ldots, U_{i_{n}}$ and $V_{j_{1}}, \ldots, V_{j_{n+1}}$ are all isomorphic to $R_{R}$. By cancelling $U_{i_{1}}, \ldots, U_{i_{n}}$ and $V_{j_{1}}, \ldots, V_{j_{n}}$ from both sides (Lemma 2.4), we get $B \cong C \oplus V_{j_{n+1}}$.

Now suppose that $U \neq R_{R}$. For any $k \in I$ and $\ell \in J$, let $\pi_{k}: \bigoplus_{i \in I} U_{i} \rightarrow U_{k}$, $p_{\ell}: \bigoplus_{j \in J} V_{j} \rightarrow V_{\ell}, \varepsilon_{k}: U_{k} \rightarrow \bigoplus_{i \in I} U_{i}$ and $e_{\ell}: V_{\ell} \rightarrow \bigoplus_{j \in J} V_{j}$ be the canonical projections and injections. Apply Lemma 3.2 to $U_{i_{1}}$ and the direct sum $M=\bigoplus_{j \in J} V_{j}$, so that there is $j \in J$ such that $\pi_{i_{1}} e_{j} p_{j} \varepsilon_{i_{1}} \notin \mathcal{K}_{U_{i_{1}}}$. By Lemma 2.6, we have $\left[U_{i_{1}}\right]_{e}=\left[V_{j}\right]_{e}$. One of the following two cases holds.

(1) $j$ is equal to some $j_{t}$, say $j_{1}$. Thus $U_{i_{1}} \cong V_{j_{1}}$ (Lemma 2.2). Therefore,

$$
U_{i_{2}} \oplus \cdots \oplus U_{i_{n}} \oplus B \cong V_{j_{2}} \oplus \cdots \oplus V_{j_{n+1}} \oplus C
$$

by Lemma 2.4 . 
(2) $j \neq j_{t}$ for every $t=1, \ldots, n+1$. Since $\left[U_{i_{1}}\right]_{l}=\left[V_{j_{1}}\right]_{l}$, by Lemma 2.3, $U_{i_{1}} \oplus U^{\prime} \cong V_{j_{1}} \oplus V_{j}$ for some cyclically presented module $U^{\prime}$. Then, again by Lemma 2.4 ,

$$
U_{i_{2}} \oplus \cdots \oplus U_{i_{n}} \oplus B \cong V_{j_{2}} \oplus \cdots \oplus V_{j_{n+1}} \oplus D
$$

for some suitable module $D$. An easy induction argument shows that after $n$ steps we get the required contradiction.

Now suppose that $I(U)$ and $J(U)$ are both infinite. By symmetry it is sufficient to prove that $|J(U)| \leq|I(U)|$. For every $k \in I(U)$, define a subset $A(k)$ of $J(U)$ by setting $A(k):=\left\{\ell \in J(U) \mid p_{\ell} \varepsilon_{k} \pi_{k} e_{\ell}\right.$ is an automorphism of $\left.V_{\ell}\right\}$ if $U \cong R_{R}$, and $A(k):=\left\{\ell \in J(U) \mid p_{\ell} \varepsilon_{k} \pi_{k} e_{\ell} \notin \mathcal{I}_{V_{\ell}}\right\}$ if $U$ is non-projective. Note that $A(k)$ is a finite set because there is a finite subset $F$ of $J$ with $U_{k} \subseteq \bigoplus_{j \in F} V_{j}$, so that $p_{\ell} \varepsilon_{k}=0$ for every $\ell \in J \backslash F$. We claim that

$$
J(U)=\bigcup_{k \in I(U)} A(k)
$$

In order to prove the claim take an index $\ell \in J(U)$. By Lemma 3.2 applied to the direct summand $V_{\ell}$ of $M=\bigoplus_{i \in I} U_{i}$, there exists an index $k \in I$ such that $p_{\ell} \varepsilon_{k} \pi_{k} e_{\ell}$ is an automorphism of $V_{\ell}$ when $U \cong R_{R}$ and $p_{\ell} \varepsilon_{k} \pi_{k} e_{\ell} \notin \mathcal{I}_{V_{\ell}}$ when $U$ is non-projective. Hence in each case we have $\left[U_{k}\right]_{l}=\left[V_{\ell}\right]_{l}=[U]_{l}$ (Lemma 2.6), i.e., $k \in I(U)$ and $\ell \in A(k)$. This proves the claim.

It follows that $|J(U)| \leq \aleph_{0}|I(U)|=|I(U)|$. Hence $|J(U)|=|I(U)|$ if $I(U)$ and $J(U)$ are both infinite. This concludes the proof of Theorem 3.1.

\section{Monoids of isomorphism classes.}

In [1], we have associated to every local ring $R$ a bipartite, non-directed graph $G=G(R)$, without multiple edges, which describes the behavior of cyclically presented right $R$-modules. It is constructed in the following way. Let $L, E$ and $I$ be sets of representatives up to having the same lower part, epigeny class and isomorphism of all non-projective cyclically presented right $R$-modules, respectively. The set of vertices of $G$ is the disjoint union of $L$ and $E$, and the set of edges of $G$ is $I$. The graph $G=(L \cup E, I)$ is bipartite because there are no edges between two vertices in $L$ or between two vertices in $E$. An edge $U \in I$ connects the vertices $V \in L$ and $W \in E$ if and only if $[U]_{l}=[V]_{l}$ and $[U]_{e}=[W]_{e}$. We identify $L, E$ and $I$ with the classes of all lower parts, all epigeny classes and all isomorphism classes, respectively, of non-projective cyclically presented right $R$-modules, so that $L=\left\{[U]_{l} \mid U\right.$ is non-projective cyclically presented $\}, E=\left\{[U]_{e} \mid U\right.$ 
is non-projective cyclically presented $\}$ and $I=\{\langle U\rangle \mid U$ is non-projective cyclically presented $\}$, and $\langle U\rangle$ is the unique edge between $[U]_{l}$ and $[U]_{e}$.

The connected components of $G$ define a partition of the set $L \cup E$ of vertices of the graph $G$ and a partition of the set $I$ of edges, and the connected components of $G$ are full subgraphs of $G$. A full subgraph of a graph $G$ without multiple edges is a subgraph $G^{\prime}$ of $G$ such that any two vertices of $G^{\prime}$ adjacent in $G$ are adjacent in $G^{\prime}$ as well. Thus a connected component of $G$ will be of the type $C=\left(L_{C} \cup E_{C}, I_{C}\right)$ for suitable subsets $L_{C}, E_{C}$ and $I_{C}$ of $L, E$ and $I$, respectively. We say that two non-projective cyclically presented right $R$-modules $U$ and $V$ are in the same connected component if there is a connected component $C$ of $G$ with $\langle U\rangle \in I_{C}$ and $\langle V\rangle \in I_{C}$.

A graph is called a complete bipartite graph if there is a partition $X \cup Y$ of its set of vertices for which $X \neq \emptyset, Y \neq \emptyset$, there are no edges between any two vertices in $X$, no edges between any two vertices in $Y$, and exactly one edge between any vertex in $X$ and any vertex in $Y$. For any local ring $R$, the connected components $C=\left(L_{C} \cup E_{C}, I_{C}\right)$ of the graph $G=(L \cup E, I)$ are complete bipartite graphs [1, Proposition 8.1].

Let $G=(V \cup W, I)$ be a bipartite graph and let $F:=\mathbb{N}_{0}^{(V)} \oplus \mathbb{N}_{0}^{(W)}$ be the free commutative monoid with free set of generators the disjoint union $V \cup W$. The elements of $F$ are tuples of nonnegative integers, almost all zero, indexed in $V \cup W$. We write the elements of $F$ in the form $\left(a_{v}\right)_{v \in V} \cup\left(b_{w}\right)_{w \in W}$. Here the $a_{v}$ 's and the $b_{w}$ 's belong to $\mathbb{N}_{0}$ and are almost all zero. Let $S_{G}$ be the submonoid of $F$ whose elements are all $\left(a_{v}\right)_{v \in V} \cup$ $\cup\left(b_{w}\right)_{w \in W} \in F$ with $\sum_{v \in V_{C}} a_{v}=\sum_{w \in W_{C}} b_{w}$ for every connected component $C=\left(V_{C} \cup W_{C}, I_{C}\right)$ of $G$. For instance, for every edge $\{\bar{v}, \bar{w}\}$ of $G$, the element $f_{\bar{v}, \bar{w}}:=\left(\delta_{\bar{v}, v}\right)_{v \in V} \cup\left(\delta_{\bar{w}, w}\right)_{w \in W} \in F$, where $\delta$ is the Kronecker delta, is in $S_{G}$. The set of all $f_{v, w}$, where $\{v, w\}$ ranges in the set of all edges of $G$, is a set of generators for the monoid $S_{G}$. Moreover, the $f_{v, w}$ 's are precisely all atoms of the monoid $S_{G}$. Recall that a non-zero element $m$ of a commutative monoid $M$ is an atom of $M$ if for every $a, b \in M, m=a+b$ implies $a=0$ or $b=0$.

Let $\mathrm{SCP}^{*}-R$ be the class of all right $R$-modules that are finite direct sums of cyclically presented modules, and SCP- $R$ the class of all right $R$ modules that are finite direct sums of cyclically presented modules nonisomorphic to $R_{R}$. For any module $M_{R}$, let $\left\langle M_{R}\right\rangle:=\left\{N_{R} \mid N_{R}\right.$ is an $R$ module isomorphic to $\left.M_{R}\right\}$ be the isomorphism class of $M_{R}$, and let $V\left(\mathrm{SCP}^{*}-R\right):=\left\{\left\langle M_{R}\right\rangle \mid M_{R} \in \mathrm{SCP}^{*}-R\right\}$ be the monoid of all isomorphism class of finite direct sums of cyclically presented $R$-modules with addition induced by direct sum: $\left\langle M_{R}\right\rangle+\left\langle N_{R}\right\rangle=\left\langle M_{R} \oplus N_{R}\right\rangle$. Similarly, consider the submonoid $V(\mathrm{SCP}-R):=\left\{\left\langle M_{R}\right\rangle \mid M_{R} \in \mathrm{SCP}-R\right\}$ of $V\left(\mathrm{SCP}^{*}-R\right)$. The fact 
that every $R$-module in $\mathrm{SCP}^{*}-R$ is uniquely of the form $P \oplus N$ with $P$ free and $N \in \mathrm{SCP}-R$ implies that $V\left(\mathrm{SCP}^{*}-R\right) \cong \mathbb{N}_{0} \oplus V(\mathrm{SCP}-R)$, where $\mathbb{N}_{0}$ corresponds to the finitely generated free modules, that is, to the cyclic submonoid of $V\left(\mathrm{SCP}^{*}-R\right)$ generated by $\left\langle R_{R}\right\rangle$.

By [1, Theorem 5.3], there is a subdirect embedding $V($ SCP- $R) \rightarrow$ $\rightarrow \mathbb{N}_{0}^{(E)} \oplus \mathbb{N}_{0}^{(L)}$. Here $E$ and $L$ are the sets of all epigeny classes and all lower parts of non-projective cyclically presented right $R$-modules. For the graph $G=(L \cup E, I)$, the monoids $V(\mathrm{SCP}-R)$ and $S_{G}$ are isomorphic [1, Theorem 8.3], so that $V\left(\mathrm{SCP}^{*}-R\right) \cong \mathbb{N}_{0} \oplus S_{G}$

As we have recalled above, for a local ring $R$, the graph $G=(L \cup E, I)$ is the disjoint union of its connected components $C$, which are complete bipartite graphs [1, Proposition 8.1]. It follows that $S_{G}=\bigoplus_{C} S_{C}$. Now a complete bipartite graph $C$ is completely determined by two cardinal numbers $\alpha \geq \beta \geq 1$. More precisely, for every pair of cardinal numbers $\alpha \geq \beta \geq 1$, let $C(\alpha, \beta)$ be the complete bipartite graph with set of vertices the disjoint union of $\alpha$ and $\beta$ and exactly one edge between each $a \in \alpha$ and each $b \in \beta$. Then, for every complete bipartite graph $C$, there is one pair $(\alpha, \beta)$ of cardinal numbers $\alpha \geq \beta \geq 1$ with $C \cong C(\alpha, \beta)$. Notice that the commutative monoid $S_{C(\alpha, \beta)}$ is a free commutative monoid if and only if $\beta=1$, and in this case $S_{C(\alpha, \beta)}$ is isomorphic to $\mathbb{N}_{0}^{(\alpha)}$. More precisely, we have the following.

Lemma 4.1. For cardinal numbers $\alpha \geq \beta \geq 1, \gamma \geq \delta \geq 1$, the monoids $S_{C(\alpha, \beta)}$ and $S_{C(\gamma, \delta)}$ are isomorphic if and only if $\alpha=\gamma$ and $\beta=\delta$.

Proof. A monoid isomorphism $S_{C(\alpha, \beta)} \rightarrow S_{C(\gamma, \delta)}$ sends the set $X$ of all atoms of $S_{C(\alpha, \beta)}$ onto the set $Y$ of all atoms of $S_{C(\gamma, \delta)}$. The atoms of $S_{C(\alpha, \beta)}$ are the elements of the form $f_{a, b}$, where $a$ ranges in $\alpha$ and $b$ ranges in $\beta$. Equivalently, $X$ corresponds to the set of all edges of the graph $C(\alpha, \beta)$. Let $\mathcal{F}$ be the family of all subsets $X^{\prime}$ of $X$ such that the divisor-closed submonoid $\left\langle X^{\prime}\right\rangle$ of $S_{C(\alpha, \beta)}$ generated by $X^{\prime}$ is a free monoid with free set of generators $X \cap\left\langle X^{\prime}\right\rangle$. Recall that the divisor-closed submonoid of $S_{C(\alpha, \beta)}$ generated by $X^{\prime}$ is the set of all elements $s \in S_{C(\alpha, \beta)}$ such that there exist $n \geq 1$, $x_{1}^{\prime}, \ldots, x_{n}^{\prime} \in X^{\prime}$ and $t \in S_{C(\alpha, \beta)}$ with $s+t=x_{1}^{\prime}+\ldots+x_{n}^{\prime}$. Notice that if $f_{a, b}$ and $f_{c, d}$ are two atoms in a subset $X^{\prime} \in \mathcal{F}$, then the two corresponding edges $\{a, b\}$ and $\{c, d\}$ are incident, because otherwise there is a relation $f_{a, b}+f_{c, d}=f_{a, d}+f_{c, b}$ between elements of $X \cap\left\langle X^{\prime}\right\rangle$. It follows that the elements $X^{\prime} \in \mathcal{F}$ correspond to subgraphs of $C(\alpha, \beta)$ that are stars. If we partially order $\mathcal{F}$ by inclusion, then the maximal elements of $\mathcal{F}$ have cardinality either $\alpha$ or $\beta$. Similarly for $S_{C(\gamma, \delta)}$. Hence the isomorphism type of 
$S_{C(\alpha, \beta)}$ determines $\alpha$ and $\beta$, that is, $S_{C(\alpha, \beta)} \cong S_{C(\gamma, \delta)}$ implies $\{\alpha, \beta\}=\{\gamma, \delta\}$. From $\alpha \geq \beta \geq 1$ and $\gamma \geq \delta \geq 1$, it now follows that $\alpha=\gamma$ and $\beta=\delta$.

Therefore $S_{G} \cong \mathbb{N}_{0}^{(\aleph)} \oplus\left(\bigoplus_{i \in I} S_{C\left(\alpha_{i}, \beta_{i}\right)}\right)$ for a cardinal number $\aleph$ and an indexed set $\left\{\left(\alpha_{i}, \beta_{i}\right) \mid i \in I\right\}$ of pairs $\left(\alpha_{i}, \beta_{i}\right)$ of cardinal numbers with $\alpha_{i} \geq \beta_{i} \geq 2$. The following proposition shows that the cardinal number $\aleph$ and the indexed set of pairs $\left(\alpha_{i}, \beta_{i}\right), i \in I$, in this direct-sum decomposition of the commutative monoid $S_{G}$ are uniquely determined by the ring $R$.

Proposition 4.2. If there is a monoid isomorphism $\Psi: \mathbb{N}_{0}^{(\aleph)} \oplus$ $\oplus\left(\bigoplus_{i \in I} S_{C\left(\alpha_{i}, \beta_{i}\right)}\right) \cong \mathbb{N}_{0}^{\left(\aleph^{\prime}\right)} \oplus\left(\bigoplus_{j \in J} S_{C\left(\alpha_{j}^{\prime}, \beta_{j}^{\prime}\right)}\right)$ for cardinal numbers $\aleph, \aleph^{\prime}$ and $\alpha_{i} \geq \beta_{i} \geq 2(i \in I)$ and $\alpha_{j}^{\prime} \geq \beta_{j}^{\prime} \geq 2(j \in J)$, then $\aleph=\aleph^{\prime}$ and there is $a$ bijection $f: I \rightarrow J$ such that $\left(\alpha_{i}, \beta_{i}\right)=\left(\alpha_{f(i)}^{\prime}, \beta_{f(i)}^{\prime}\right)$ for every $i \in I$.

Proof. The monoid isomorphism $\Psi$ sends the set of atoms onto the set of atoms. Since there are no nontrivial relations between the atoms contained in $\mathbb{N}_{0}^{(\aleph)}$ and every atom in $\left(\bigoplus_{i \in I} S_{C\left(\alpha_{i}, \beta_{i}\right)}\right)$ is subject to some nontrivial relation because $\alpha_{i} \geq \beta_{i} \geq 2$, it follows that $\aleph=\aleph^{\prime}$ and that the monoid isomorphism $\Psi$ restricts to an isomorphism $\bigoplus_{i \in I} S_{C\left(\alpha_{i}, \beta_{i}\right)} \cong \bigoplus_{j \in J} S_{C\left(\alpha_{j}^{\prime}, \beta_{j}^{\prime}\right)}$. We show that for any $i \in I$, there is a unique $j \in J$ with $\Psi\left(S_{C\left(\alpha_{i}, \beta_{i}\right)}\right) \subseteq S_{C\left(\alpha_{j}^{\prime}, \beta_{j}^{\prime}\right)}$. By considering the same property for $\Psi^{-1}$, we conclude that $\Psi$ restricts to an isomorphism $S_{C\left(\alpha_{i}, \beta_{i}\right)} \cong S_{C\left(\alpha_{j}^{\prime}, \beta_{j}^{\prime}\right)}$. Thus by Lemma $4.1, \alpha_{i}=\alpha_{j}^{\prime}$ and $\beta_{i}=\beta_{j}^{\prime}$.

For a fixed $i \in I$, let $a, a^{\prime} \in \alpha_{i}$ and $b, b^{\prime} \in \beta_{i}$ be distinct elements. There exists $j \in J$ such that $\Psi\left(f_{a, b}\right) \in S_{C\left(\alpha_{j}^{\prime}, \beta_{j}^{\prime}\right)}$. Since $f_{a, b}+f_{a^{\prime}, b^{\prime}}=f_{a, b^{\prime}}+f_{a^{\prime}, b}$, we infer that $\Psi\left(f_{a, b}\right), \Psi\left(f_{a, b^{\prime}}\right), \Psi\left(f_{a^{\prime}, b}\right)$ and $\Psi\left(f_{a^{\prime}, b^{\prime}}\right)$ must be in the same component, i.e., in $S_{C\left(\alpha_{j}^{\prime}, \beta_{j}^{\prime}\right)}$. Now for any $a^{\prime \prime} \in \alpha_{i}$ and $b^{\prime \prime} \in \beta_{i}$, there is $(x, y) \in$ $\in\left\{(a, b),\left(a, b^{\prime}\right),\left(a^{\prime}, b\right),\left(a^{\prime}, b^{\prime}\right)\right\}$ such that $x \neq a^{\prime \prime}$ and $y \neq b^{\prime \prime}$. Again from the relation $f_{x, y}+f_{a^{\prime \prime}, b^{\prime \prime}}=f_{x, b^{\prime \prime}}+f_{a^{\prime \prime}, y}$ we conclude that $\Psi\left(f_{a^{\prime \prime}, b^{\prime \prime}}\right) \in S_{C\left(\alpha_{j}^{\prime}, \beta_{j}^{\prime}\right)}$ because $\Psi\left(f_{x, y}\right) \in S_{C\left(\alpha_{j}^{\prime}, \beta_{j}^{\prime}\right)}$. Therefore, $\Psi\left(S_{C\left(\alpha_{i}, \beta_{i}\right)}\right) \subseteq S_{C\left(\alpha_{j}^{\prime}, \beta_{j}^{\prime}\right)}$, as desired.

REMARK 4.3. (a) The previous results in this Section hold not only for the graph that describes the cyclically presented modules over a local ring, which we have introduced in this paper, but also for the graph that describes the uniserial modules over an arbitrary ring, which Př́ihoda and the third author introduced in [6, 7]. The following fact, on the contrary, applies only to our graph and not to that introduced in [6, 7]. Let $R$ be a local ring and $G=(L \cup E, I)$ be our graph. Here $I$ is the set of all isomorphism classes of non-projective cyclically presented right $R$-modules. Let $J^{*}(R)$ 
be the set $J(R) \backslash\{0\}$. Recall that two cyclically presented right $R$-modules $R / a R, R / b R\left(a, b \in J^{*}(R)\right)$ are isomorphic if and only if there exist $u, v \in U(R)$ with $a=u b v$ [1, Lemma 2.4]. Thus there is a right action of the group $U(R)^{o p} \times U(R)$ on the set $J^{*}(R)$, and $I$ turns out to be the set of all orbits $(a):=U(R) a U(R)$ with respect to this group action. With this point of view, $E$, the set of all epigeny classes of non-projective cyclically presented right $R$-modules, is the quotient set of $I=\left\{(a) \mid a \in J^{*}(R)\right\}$ with respect to the equivalence relation $\sim$ on $I$ defined by $(a) \sim(b)$ if $[R / a R]_{e}=[R / b R]_{e}$, that is, if and only if there exist $u, v \in U(R)$ and $r, s \in R$ with $u a=b r$ and $v b=a s$. Similarly, $L$ turns out to be the quotient set of $I$ with respect to the equivalence relation $\equiv$ on $I$ defined by $(a) \equiv(b)$ if $[R / a R]_{l}=[R / b R]_{l}$, that is, if and only if there exist $u, v \in U(R)$ and $r, s \in R$ with $a u=r b$ and $b v=s a$.

(b) The graph $G(R)$ that describes cyclically presented modules over a local ring $R$ is the same for right modules and left modules, that is, it is a left/right symmetric invariant of $R$.

Clearly, the graph $G=G(R)$ and the monoid $S_{G}$ describe the behavior of finite direct-sums of cyclically presented modules over a local ring $R$, but in view of our Theorem 3.1 the constructions of the graph $G$ and the monoid $S_{G}$ can be extended to arbitrary cardinalities in the following way.

Fix a local ring $R$ and an infinite cardinal number $\aleph$. Let $\mathrm{SCP}_{\aleph}^{*} R$ be the class of all right $R$-modules that are direct sums of families of cardinality $<\aleph$ of cyclically presented $R$-modules, and $\mathrm{SCP}_{\aleph}-R$ the class of all right $R$ modules that are direct sums of families of cardinality $<\aleph$ of cyclically presented modules non-isomorphic to $R_{R}$. Thus SCP- $R=\mathrm{SCP}_{\aleph_{0}}-R$ and SCP $R=\mathrm{SCP}_{\aleph_{0}}^{*}-R$. Let $V_{\aleph}^{*}:=V\left(\mathrm{SCP}_{\aleph}^{*}-R\right)$ and $V_{\aleph}:=V\left(\mathrm{SCP}_{\aleph}-R\right)$ be the corresponding monoids of all isomorphism classes of modules in $\mathrm{SCP}_{\aleph}^{*}-R$ and $\mathrm{SCP}_{\aleph}-R$, respectively. Thus $V_{\aleph}^{*}=\left\{\left\langle\bigoplus_{i \in I} R / a_{i} R\right\rangle:|I|<\aleph, a_{i} \in R\right\}$ and $V_{\aleph}=\left\{\left\langle\bigoplus_{i \in I} R / a_{i} R\right\rangle:|I|<\aleph, a_{i} \in R \backslash\{0\}\right\}$.

LEMma 4.4. Every $R$-module in $\mathrm{SCP}_{\aleph}^{*}-R$ is uniquely of the form $P \oplus N$ with $P$ free and $N \in \mathrm{SCP}_{\aleph}-R$, that is, if $P \oplus N \cong P^{\prime} \oplus N^{\prime}$ with $P, P^{\prime}$ free and $N, N^{\prime}$ in $\mathrm{SCP}_{\aleph}-R$, then $P \cong P^{\prime}$ and $N \cong N^{\prime}$.

Proof. Suppose that $P=\bigoplus_{i \in I} U_{i}$ and $P^{\prime}=\bigoplus_{j \in J} U_{j}^{\prime}$, where $U_{i} \cong U_{j}^{\prime} \cong R_{R}$ for every $i \in I$ and $j \in J$. Also suppose that $N=\bigoplus_{k \in K} V_{k}$ and $N^{\prime}=\bigoplus_{l \in L} V_{l}^{\prime}$, where the $V_{k}$ 's and the $V_{l}^{\prime}$ 's are non-projective cyclically presented modules and $P \oplus N \cong P^{\prime} \oplus N^{\prime}$. An application of Theorem 3.1 to the isomorphism 
$P \oplus N \cong P^{\prime} \oplus N^{\prime}$ gives that there are two bijections $\sigma, \tau: I \cup K \rightarrow J \cup L$ corresponding to the lower parts and epigeny classes. Since a non-projective cyclically presented module does not have the same lower part or epigeny class as $R_{R}, \sigma$ and $\tau$ induce bijections $I \rightarrow J$ and $K \rightarrow L$. Now again by Theorem 3.1, we have $P \cong P^{\prime}$ and $N \cong N^{\prime}$.

From Lemma 4.4, it follows that $V_{\aleph}^{*} \cong\left[0, \aleph\left[\oplus V_{\aleph}\right.\right.$, where $[0, \aleph[$ is the commutative additive monoid of all cardinal numbers $<\aleph$.

For an arbitrary bipartite graph $G=(V \cup W, I)$ and an infinite cardinal $\aleph$, let $P:=\left[0, \aleph\left[{ }^{V} \times\left[0, \aleph\left[{ }^{W}\right.\right.\right.\right.$ be the direct product of a family of copies of the additive monoid $[0, \aleph[$ whose cardinality is equal to the cardinality of the disjoint union $V \cup W$. The elements of $P$ are tuples of cardinals $<\aleph$ indexed in $V \cup W$. Write the elements of $P$ in the form $\left(\alpha_{v}\right)_{v \in V} \cup\left(\beta_{w}\right)_{w \in W}$, where the $\alpha_{v}$ 's and the $\beta_{w}$ 's are cardinals $<\aleph$. Let $S_{G, \aleph}$ be the submonoid of $P$ whose elements are all $\left(\alpha_{v}\right)_{v \in V} \cup\left(\beta_{w}\right)_{w \in W} \in P$ subject to two conditions: (1) $\sum_{v \in V_{C}} \alpha_{v}=\sum_{w \in W_{C}} \beta_{w}$ for every connected component $C=\left(V_{C} \cup W_{C}, I_{C}\right)$ of $G$; (2) $\sum_{v \in V} \alpha_{v}<\aleph$. Notice that the sum of any set of cardinals is defined as the cardinality of their disjoint union.

THEOREM 4.5. For the graph $G=(L \cup E, I)$ of a local ring $R$, the monoids $V_{\aleph}$ and $S_{G, \aleph}$ are isomorphic. Thus $V_{\aleph}^{*} \cong\left[0, \aleph\left[\oplus S_{G, \aleph}\right.\right.$.

Proof. By Theorem 3.1, there is a well-defined subdirect embedding $\Phi: V_{\aleph} \rightarrow P=\left[0, \aleph\left[{ }^{L} \times\left[0, \aleph\left[{ }^{E}\right.\right.\right.\right.$ defined as follows. Let $M=\bigoplus_{j \in J} U_{j} \in \mathrm{SCP}_{\aleph^{-}}$ $R$. For any non-projective cyclically presented module $U$ let $L_{U}(M)=$ $=\left\{j \in J:\left[U_{j}\right]_{l}=[U]_{l}\right\}$ and $E_{U}(M)=\left\{j \in J:\left[U_{j}\right]_{e}=[U]_{e}\right\}$. Then the $E_{U}(M)$ 's and the $L_{U}(M)$ 's form two partitions of $J$. Define

$$
\Phi(\langle M\rangle)=\left(\left|L_{U}(M)\right|\right)_{[U]_{l} \in L} \cup\left(\left|E_{U}(M)\right|\right)_{[U]_{e} \in E} .
$$

Hence it suffices to show that the image of $\Phi$ is $S_{G, \aleph}$. Now if $A=\bigoplus_{j \in J} R / a_{j} R$ is a direct sum of non-projective cyclically presented modules $R / a_{j} R(|J|<\aleph)$, the partition of $G$ into its connected components $C$ induces a partition $\left\{J_{C} \mid C\right.$ is a connected component of $\left.G\right\}$ of $J$, so that $A=\bigoplus_{C}\left(\bigoplus_{j \in J_{C}} R / a_{j} R\right)$. Thus if $\Phi(\langle A\rangle)=\left(\alpha_{l}\right)_{l \in L} \cup\left(\beta_{e}\right)_{e \in E}$, then, for every connected component $C$ of

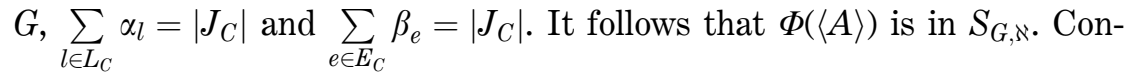
versely, let $\left(\alpha_{l}\right)_{l \in L} \cup\left(\beta_{e}\right)_{e \in E}$ be an element of $S_{G, \aleph}$. For every connected component $C$ of $G, \sum_{l \in L_{C}} \alpha_{l}=\sum_{e \in E_{C}} \beta_{e}$ by Condition (1), so that there exists a 
set $J_{C}$ of cardinality $\left|J_{C}\right|=\sum_{l \in L_{C}} \alpha_{l}=\sum_{e \in E_{C}} \beta_{e}$ with two mappings $\lambda_{C}: J_{C} \rightarrow L_{C}$ and $\eta_{C}: J_{C} \rightarrow E_{C}$ such that for every $l \in L_{C}$ the inverse image of $l$ in $J_{C}$ via $\lambda_{C}$ has cardinality $\alpha_{l}$, and for every $e \in E_{C}$ the inverse image of $e$ in $J_{C}$ via $\eta_{C}$ has cardinality $\beta_{e}$ (this follows immediately from the definition of sum of a set of cardinals recalled above). Since the connected component $C$ is a complete bipartite graph, for every $j \in J_{C}$ there is a non-projective cyclically presented module $R / a_{j} R$ with epigeny class $\eta_{C}(j)$ and with lower part $\lambda_{C}(j)$. Then $A:=\bigoplus_{C}\left(\bigoplus_{j \in J_{C}} R / a_{j} R\right)$ is in $\operatorname{SCP}_{\aleph}-R$ by Condition (2), and $\Phi(\langle A\rangle)=\left(\alpha_{l}\right)_{l \in L} \cup\left(\beta_{e}\right)_{e \in E}$.

\section{REFERENCES}

[1] B. Amini - A. Amini - A. Facchini, Equivalence of diagonal matrices over local rings, J. Algebra, 320 (2008), pp. 1288-1310.

[2] F. W. Anderson - K. R. Fuller, Rings and Categories of Modules, Second edition, GTM 13 (Springer-Verlag, New York, 1992).

[3] N. V. Dung - A. Facchini, Weak Krull-Schmidt for infinite direct sums of uniserial modules, J. Algebra, 193 (1997), pp. 102-121.

[4] A. Facchini, Krull-Schmidt fails for serial modules, Trans. Amer. Math. Soc., 348 (1996), pp. 4561-4575.

[5] A. Facchini, Module Theory. Endomorphism rings and direct sum decompositions in some classes of modules, Progress in Math., 167 (Birkhäuser Verlag, Basel, 1998).

[6] A. FACCHINI - P. Př́íHodA, Monogeny dimension relative to a fixed uniform module, J. Pure Appl. Algebra, 212, no. 9 (2008), pp. 2092-2104.

[7] A. FACCHINI - P. Př́íHODA, Representations of the category of serial modules of finite Goldie dimension, in "Models, Modules, and Abelian Groups", R. Göbel and B. Goldsmith Eds., de Gruyter (Berlin-New York, 2008), pp. 463-486.

[8] P. PŘíHoDA, Weak Krull-Schmidt theorem and direct sum decompositions of serial modules offinite Goldie dimension, J.Algebra 281, no. 1(2004), pp. 332-341.

[9] P. P̌̌íHodA, A version of the weak Krull-Schmidt theorem for infinite direct sums of uniserial modules, Comm. Algebra, 34, no. 4 (2006), pp. 1479-1487.

[10] G. Puninski, Some model theory over an exceptional uniserial ring and decompositions of serial modules, J. London Math. Soc., 64, no. 2 (2001), pp. $311-326$.

[11] G. Puninski, Some model theory over a nearly simple uniserial domain and decompositions of serial modules, J. Pure Appl. Algebra, 163, no. 3 (2001), pp. $319-337$.

[12] R. B. WARFIELD, JR., Purity and algebraic compactness for modules, Pacific J. Math., 28 (1969), pp. 699-719.

Manoscritto pervenuto in redazione il 3 giugno 2008. 